\title{
Total neoadjuvant therapy of locally advanced rectal cancer: Neoadjuvant FOLFOXIRI chemotherapy followed by short-course radiation and consolidated XELOX chemotherapy prior to resection
}

\author{
Yuanhe Wang ${ }^{1 *}$, Mingyi Zhou ${ }^{2 *}$ and Jingdong Zhang ${ }^{1 *}$ \\ ${ }^{1}$ Department of Gastrointestinal Medical Oncology, Cancer Hospital of China Medical University, Liaoning Cancer Hospital \& Institute, Shenyang 110042, \\ Liaoning Province, PR China \\ ${ }^{2}$ Department of Gynecology, Cancer Hospital of China Medical University, Liaoning Cancer Hospital \& Institute, Shenyang 110042, Liaoning Province, PR China \\ \#These authors contributed equally to this work
}

Rectal cancer is a common cancer worldwide. According to the 2017 cancer statistics in US, rectal cancer has become $3^{\text {rd }}$ reason for new cases and $2^{\text {nd }}$ reason for deaths in male patients and $3^{\text {rd }}$ reason for death in female patients. There were an estimated 39,910 new cases in the United States in 2017 [1].

Locally advanced rectal cancer (LARC) consists of stage II (T3/4, node-negative, M0) and stage III (T3/4, node-positive, M0) rectal cancer. The rectal cancer located in the mid-low rectum (up to $12 \mathrm{~cm}$ from anal verge) was identified to penetrate through the muscle wall with or without nodal metastasis by imaging or pathological test (c/ pT3-4b, or c/pN1-2) but without distal metastases (M0). The risk of pelvic recurrence of rectal cancer (especially for LARC) is higher than colon cancer. And the local recurrence of rectal cancer could lead to a poor prognosis [2]. The high risk of locoregional recurrence is associated with the unique anatomy of rectum: close to pelvic organs and structures, absent a serosa surrounding the rectum, and difficult to obtain wide surgical margins at resection. The anatomy of rectum increases the technical difficulty, postoperative complication and local recurrence.

The treatment of LARC patients evolved from surgery alone to postoperative adjuvant therapy, to neoadjuvant chemoradiation therapy (chemoRT) followed by total mesorectal excision (TME) and postoperative adjuvant chemotherapy, and to total neoadjuvant therapy (TNT) followed by TME. Recent standard treatment for LARC consists of neoadjuvant chemoRT followed by TME and postoperative adjuvant chemotherapy. For adjuvant chemotherapy, according to the long-term results of ADORE trial, adjuvant FOLFOX (5-fluorouracil, oxaliplatin, leucovorin) prolonged disease free survival (DFS) than FL (5-fluorouracil, leucovorin) [3]. Preoperative radiation therapy (RT) led to less toxicities and increased compliance than postoperative RT. For preoperative RT, there are two major kinds of approaches: long-course RT (45-50 Gy in 25-28 fractions) and short-course RT (25Gy over 5 days). Combined chemotherapy and long-course RT are recommended as primary neoadjuvant chemoRT approach in the US. TME is often performed at about six weeks after neoadjuvant chemoRT. This pattern did not achieve the benefit of long-term survival, which might be due to the delay and weak dose intensity of systematic chemotherapy. Another approach of short-course RT was assessed in several European studies
[4,5]. The randomized clinical trials (RCTs) from Poland and Australian/ New Zealand demonstrated that no difference in local recurrence and overall survival (OS) between two approaches [6]. Furthermore, a randomized study that compared short-course RT followed by 3 cycles of FOLFOX chemotherapy with long-course chemoRT revealed a higher pathologic complete response (pCR) in the short-course RT group [7]. A multicenter trial suggested that consolidation mFOLFOX6 after short-course RT and lengthening the RT-to-TME increased the rates of pCR in LARC patients [8]. And the updated data of this trial suggested that consolidation mFOLFOX6 also improved the DFS [9]. These data suggested that short-course RT followed by double cytotoxic agents may be the proper approach to achieve pCR.

Neoadjuvant chemoRT followed by TME and adjuvant chemotherapy, as the primary recommended strategy, still has limitations in treating LARC patients. For one thing, the reduction of local recurrence did not convert to a longer OS. For another, the preservation of sphincter function remains an issue. According to the results of a Cochrane review of 6 RCTs, for LARC patients, neoadjuvant chemoRT reduced the risk of local recurrence, but did not improve OS, preserve sphincter function and reduce late toxicity.

To prolong OS and preserve sphincter function become the object of treating LARC patients. Considering the various neoadjuvant therapy patterns which are evaluated in the worldwide, TNT achieved the highest recommendation. TNT means all of the chemotherapy and radiation therapy were addressed prior to surgery. The data of studies showed that TNT possibly increased the rates of pCR and downstaging, facilitated resection, improved the compliance, minimized the length of time patients need an ileostomy, in-vivo assessed chemosensitivity and eradicated possible micrometastases through earlier introduction of systemic chemotherapy [10]. Furthermore, double cytotoxic

${ }^{\star}$ Correspondence to: Jingdong Zhang, Department of Gastrointestinal Medical Oncology, Liaoning Cancer Hospital \& Institute, No.44 Xiaoheyan Road, Dadong District, Shenyang 110042, Liaoning Province, PR China, Tel: +86-2431916682; Fax: +86-24-31916682; E-mail: jdzhang@cancerhosp-ln-cmu.com

Received: November 09, 2018; Accepted: December 14, 2018; Published: December 20, 2018 
Wang Y (2018) Total neoadjuvant therapy of locally advanced rectal cancer: Neoadjuvant FOLFOXIRI chemotherapy followed by short-course radiation and consolidated XELOX chemotherapy prior to resection

chemotherapy might be not enough to achieve pCR. And comparing with double cytotoxic chemotherapy, triple cytotoxic agents FOLFOXIRI (5-fluorouracil, oxaliplatin, leucovorin, irinotecan) increased ORR (objective response rate) for advanced or metastatic disease $[11,12]$. So triple cytotoxic agents FOLFOXIRI could be considered as neoadjuvant chemotherapy. The patients with remarkable downstaging may have the chance to achieve transanal local excision with a negative $(>3 \mathrm{~mm})$ margin. Patients could also maintain anal sphincter function through transanal local excision. But transanal local excision is only appropriate for selected T1, N0 early stage cancers. In other conditions, the patients who did not meet the criteria of transanal local excision could also achieve sphincter preservation function through the creation of an anastomosis after TNT.

The aim of our clinical trial (NCT03484221) is to find the best strategy to prolong long-term survival and preserve sphincter function of LARC patients. We chose triple cytotoxic agents FOLFOXIRI as the neoadjuvant chemotherapy. We will evaluate the efficacy and safety of totally neoadjuvant FOLFOXIRI chemotherapy followed by short-course radiation and XELOX chemotherapy (capecitabine and oxaliplatin) prior to TME in the patients with LARC to achieve more $\mathrm{pCR}$, longer survival and preserve sphincter function.

In this prospective study, 30 patients with LARC will be recruited. Firstly, 4 cycles of neoadjuvant FOLFOXIRI chemotherapy were administered. Subsequently, a short-course radiation therapy $\left(5 \mathrm{~Gy}^{\star} 5\right)$ will be performed. After that, 4 cycles of XELOX chemotherapy will be administered followed by TME. PET-CT examination will be performed before and after the 4 cycles of neoadjuvant FOLFOXIRI chemotherapy to assess the SUVmax changes. In addition, the dynamic changes of circulating tumor DNA (ctDNA) in peripheral blood will be monitored at the PET-CT examination. In the course of treatment, safety evaluation will be carried out according to the standard of adverse reaction classification (CTCAE) 4.0. In this trial, we address 4 cycles of neoadjuvant FOLFOXIRI chemotherapy to improve the local and systematic control. After FOLFOXIRI chemotherapy, we choose short-course RT rather than long-course RT to strengthen local control and shorten the delay of systematic therapy. Through 4 cycles of XELOX chemotherapy prior to resection, we consolidate the efficacy of systematic therapy and increase the rates of pCR after RT by lengthening the RT-to-resection. The total treatment time will be about 6 months, which is shorter than the presently standard therapy. Through TNT pattern, we aim to increase the rates of $\mathrm{pCR}$ and downstaging and improve the preservation of sphincter function. Through increasing systematic dose intensity (neoadjuvant FOLFOXIRI chemotherapy and consolidated XELOX chemotherapy after RT), we aim to improve OS and the preservation of sphincter function.

There is still limitation in our study protocol. Firstly, some patients could not tolerate FOLFOXIRI chemotherapy. We will select the appropriate patients according to the discussion of multidisciplinary team (MDT). Secondly, the patients with initial T3/4 LARC and a remarkable downstaging to $\mathrm{T} 1$ achieved the similar survival with the patients with initial T1 tumor. For these patients, consolidated XELOX chemotherapy after RT might be not necessary. So, the discussion of MDT is important for these patients. Thirdly, we acquire all the patients to address 4 cycles of XELOX chemotherapy before TME, but some patients may be adequate to achieve TME with sphincter preservation.

In conclusion, we launch a clinical trial to find the best strategy to prolong long-term survival and preserve sphincter function of LARC patients.

\section{References}

1. Siegel RL, Miller KD, Jemal A (2017) Cancer Statistics, 2017. CA Cancer J Clin 67: 7-30. [Crossref]

2. Wiig JN, Larsen SG, Giercksky KE (2005) Operative treatment of locally recurrent rectal cancer. Recent Results Cancer Res 165: 136-47. [Crossref]

3. Hong YS, Nam BH, Kim KP, Kim JE, Park SJ (2014) Oxaliplatin, fluorouracil, and leucovorin versus fluorouracil and leucovorin as adjuvant chemotherapy for locally advanced rectal cancer after preoperative chemoradiotherapy (ADORE): an openlabel, multicentre, phase 2, randomised controlled trial. Lancet Oncol 15: 1245-1253. [Crossref]

4. Swedish Rectal Cancer Trial, Cedermark B, Dahlberg M, Glimelius B, Påhlman L, et al. (1997) Improved survival with preoperative radiotherapy in resectable rectal cancer. N Engl J Med 336: 980-987. [Crossref]

5. Siegel R, Burock S, Wernecke KD, Kretzschmar A, Dietel M, et al. (2009) Preoperative short-course radiotherapy versus combined radiochemotherapy in locally advanced rectal cancer: a multi-centre prospectively randomised study of the Berlin Cancer Society. BMC Cancer 9: 50. [Crossref]

6. Bujko K, Nowacki MP, Nasierowska-Guttmejer A, Michalski W, Bebenek M, et al (2006) Long-term results of a randomized trial comparing preoperative short-course radiotherapy with preoperative conventionally fractionated chemoradiation for rectal cancer. Br J Surg 93: 1215-1223. [Crossref]

7. Bujko K, Nasierowska-Guttmejer A, Wyrwicz L, Malinowska M, Krynski J, et al (2013) Neoadjuvant treatment for unresectable rectal cancer: an interim analysis of a multicentre randomized study. Radiother Oncol 107: 171-177. [Crossref]

8. Garcia-Aguilar J, Chow OS, Smith DD, Marcet JE, Cataldo PA, et al. (2015) Effect of adding mFOLFOX6 after neoadjuvant chemoradiation in locally advanced rectal cancer: a multicentre, phase 2 trial. Lancet Oncol 16: 957-966. [Crossref]

9. Marco MR, Zhou L, Patil S, Marcet JE, Varma MG (2018) Consolidation mFOLFOX6 Chemotherapy After Chemoradiotherapy Improves Survival in Patients with Locally Advanced Rectal Cancer: Final Results of a Multicenter Phase II Trial. Dis Colon Rectum 61(10): p. 1146-1155. [Crossref]

10. Cercek A, Roxburgh CSD, Strombom P, Smith JJ, Temple LKF, et al. (2018) Adoption of Total Neoadjuvant Therapy for Locally Advanced Rectal Cancer. JAMA Oncol 4: e180071. [Crossref]

11. Gruenberger T (2015) Bevacizumab plus mFOLFOX-6 or FOLFOXIRI in patients with initially unresectable liver metastases from colorectal cancer: the OLIVIA multinational randomised phase II trial. Ann Oncol 26: 702-708. [Crossref]

12. Loupakis F, Cremolini C, Masi G, Lonardi S, Zagonel V, et al. (2014) Initial therapy with FOLFOXIRI and bevacizumab for metastatic colorectal cancer. $N$ Engl $J$ Med 371: 1609-1618. [Crossref]

Copyright: (C2018 Wang Y. This is an open-access article distributed under the terms of the Creative Commons Attribution License, which permits unrestricted use, distribution, and reproduction in any medium, provided the original author and source are credited. 